\title{
An Eddy Current Method to Evaluate Local Wall Thinning of Carbon Steel Pipe
}

\author{
Shanshan SUN ${ }^{\mathrm{a}}$, Deqianga ZHOU ${ }^{\mathrm{a}, \mathrm{b}, 1}$, Noritaka YUSA ${ }^{\mathrm{c}}$, Haicheng SONG ${ }^{\mathrm{c}}$ \\ ${ }^{a}$ School of Mechanical Engineering, Jiangnan University, Jiangsu, China \\ ${ }^{\mathrm{b}}$ Key laboratory of nondestructive testing of Nanchang Hangkong University, China \\ ${ }^{\mathrm{c}}$ Department of quantum science and energy engineering, Tohoku University, Japan
}

\begin{abstract}
This paper proposes to evaluate the local wall thinning of carbon steel pipe using an eddy current method. Firstly, the feature signals are determined by correlation analysis of the signals and the wall thinning sizes. Subsequently, the models for estimating the residual wall thickness rt is constructed using Gaussian process regression (GPR). Finally, the applicability of the models to the evaluation of local wall thinning is verified by simulation and experiment.
\end{abstract}

Keywords. local wall thinning, eddy current, correlation, Gaussian process regression

\section{Introduction}

Carbon steel pipe is widely used in industrial equipment because of their high welding ability and low price, but the wall is thinned due to flow accelerated corrosion and liquid droplet impingement [1-2]. At present, some non-destructive testing methods have been proposed, such as eddy current testing[3-6], which is an easy to implement and fast nondestructive testing technology.

This study proposes the use of eddy current method to obtain various types of signals at different frequencies to detect local wall thinning. Then the regression model for evaluating local wall thinning is established based on the correlation analysis between signals and defects. The model can evaluate the size of defects.

\section{Numerical simulation}

\subsection{Finite element model}

Numerical simulations are performed using simulation software COMSOL Multiphysics $5.2 \mathrm{a}+\mathrm{AC} / \mathrm{DC}$ module. The governing equation is:

$$
\nabla \times\left(\nabla \times \mu^{-1} A\right)+\left(j \omega \sigma-\omega^{2} \varepsilon\right) A=J_{e}
$$

\footnotetext{
${ }^{1}$ Corresponding Author, Deqiang Zhou, Associate Professor, Jiangnan University, Engaged in nondestructive testing and automation research, The Chinese, E-mail: zhoudeqiang@jiangnan.edu.cn.
} 
Where $\omega$ is angular frequency, $\varepsilon$ is permittivity, $\sigma$ is electric conductivity, $\mu$ is magnetic permeability, $A$ is magnetic vector potential . The conductivity and relative permeability of the pipe are $5.2 \mathrm{Ms} / \mathrm{m}, 160$, respectively. The current density $J_{e}$ of the excitation coil is $2.94 \times 105 \mathrm{~A} / \mathrm{m}^{2}$. Figure 1 illustrates schematic of numerical simulation.

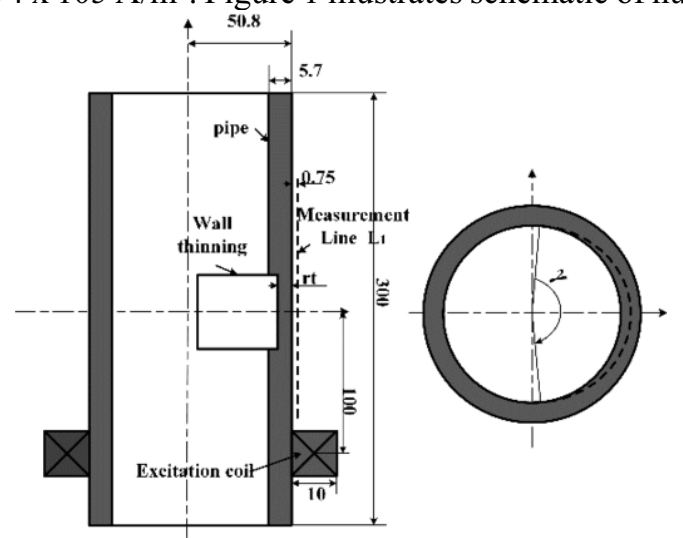

Figure 1. Schematic of numerical simulation.
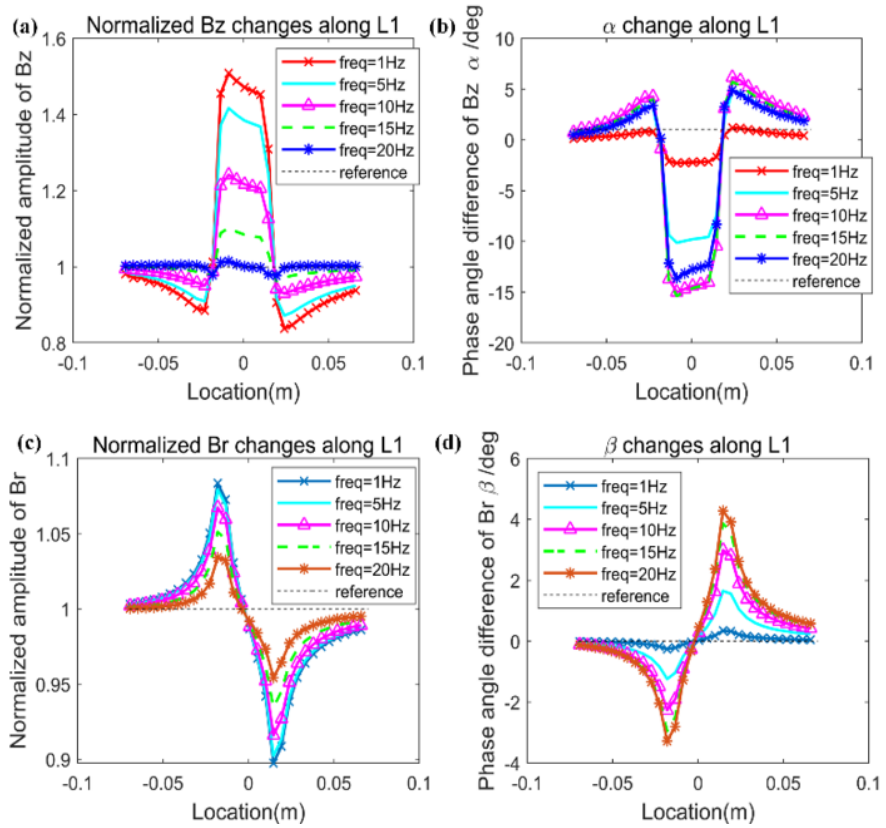

Figure 2. The change of signals along $\mathrm{L}_{1}$.

\subsection{Signal analysis}

In this paper, $1 \mathrm{~Hz}, 5 \mathrm{~Hz}, 10 \mathrm{~Hz}, 15 \mathrm{~Hz}$ and $20 \mathrm{~Hz}$ are used as the excitation frequency in order to evaluate the wall. Figure 2 shows the changes of normalized amplitude and phase difference along $\mathrm{L}_{1}$ when the pipe is thinned, and the amplitude and phase of signals are abrupt and the amplitude and phase of $\mathrm{Br}$ appear peaks and troughs at the defect. Therefore, the extreme value of the signal on $\mathrm{L}_{1}$ can be extracted as features of defect 
signals, which are denoted as $\mathrm{Bz}_{\text {maxi }}, \mathrm{Br}_{\text {maxi }}, \mathrm{Br}_{\text {mini }}, \alpha_{\text {mini }}, \beta_{\text {maxi }}, \beta_{\max }$, respectively. The subscript maxi and mini represent the maximum or minimum value of the signal on $\mathrm{L}_{1}$ at $\mathrm{iHz}$.

\subsection{Correlation analysis}

Correlation analysis is performed to determine the corresponding estimated defect size rt feature signals. Figure 3 shows that the correlation coefficients between the extreme value of the signals and the residual wall thickness $\mathrm{rt}$ is greater than 0.75 , so these extreme values can be used as the feature signals for evaluating rt. The arrows in the figure indicate the largest correlation coefficient in each type of signal. These signals are $\mathrm{Bz}_{\max 1}, \alpha_{\min 10}, \mathrm{Br}_{\min 1}, \beta_{\min 20}$, respectively.

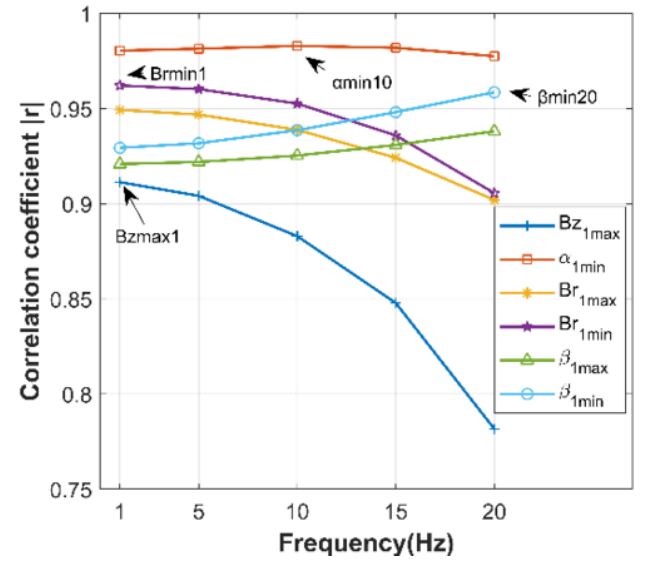

Figure 3. Correlation analysis of feature signals with rt.

\subsection{Evaluation of residual wall thickness rt by Gaussian process regression}

The Gaussian process has a strict theoretical foundation of statistical learning and has good adaptability for dealing with high-dimensional nonlinear small sample data [7]. According to the previous correlation analysis, the feature signals $\mathrm{Bz} z_{\max }, \alpha_{\min }, \mathrm{Br}_{\text {maxi }}$, $\mathrm{Br}_{\text {mini }}, \beta_{\text {maxi }}$ and $\beta_{\text {mini }}$ are highly correlated with rt. Assume that they satisfy them relationship:

$$
y=f(X)+\varepsilon
$$

Where $\mathrm{y}$ is the vector of residual wall thickness rt, $\mathrm{X}$ is the feature signals matrix, $\varepsilon$ is Gaussian noise, and the posterior distribution of $\mathrm{f}(\mathrm{X})$ is obtained from the data set $\left\{\mathrm{x}_{\mathrm{i}}\right.$, $\mathrm{y}_{\mathrm{i}}$ \} by Gaussian process . $\mathrm{y} \sim \mathrm{GP}(\mathrm{m}(\mathrm{X}), \kappa(\mathrm{X}, \mathrm{X}))$, where $\mathrm{m}(\mathrm{X})$ is the mean function, $\kappa(\mathrm{X}, \mathrm{X})$ is the covariance function, and the squared exponential kernel is expressed as:

$$
\kappa\left(x_{p}, x_{q}\right)=\sigma_{f}^{2} \exp \left[-\frac{1}{2} \frac{\left(x_{p}-x_{q}\right)^{T}\left(x_{p}-x_{q}\right)}{\sigma_{l}^{2}}\right]
$$

Where $\sigma_{\mathrm{f}}$ is the signal standard deviation and $\sigma_{1}$ is the characteristic length scale. 
Figure 4 shows the evaluation effect of the model when different signal combinations are used as predictors. As shown in Figure 4-(f), the RMSE=0.016, which is the smallest, the evaluated value of $\mathrm{rt}$ is the closest to the true value. This result corresponds to the previous correlation analysis, and the selection of signals with high correlation coefficient as predictors will improve the evaluation effect of the model. predictor: $\mathrm{Bz}_{\max i}$
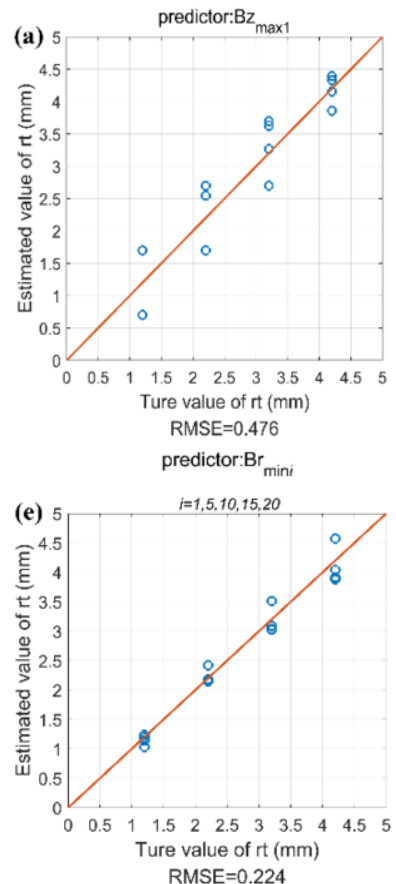
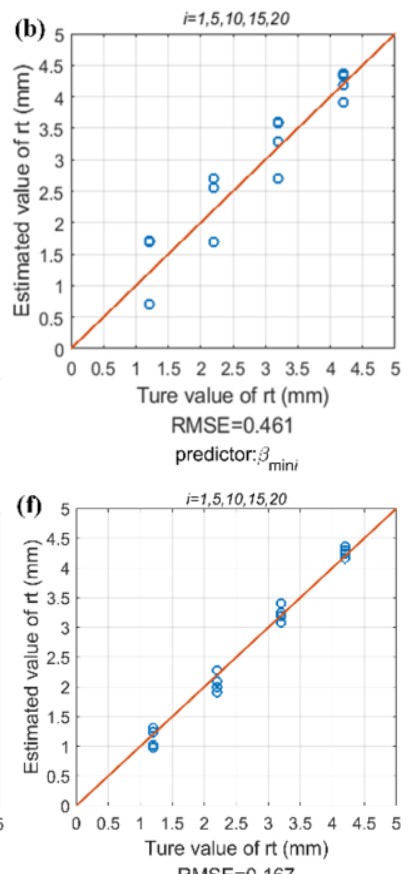

predictor: $\alpha_{\min i}$
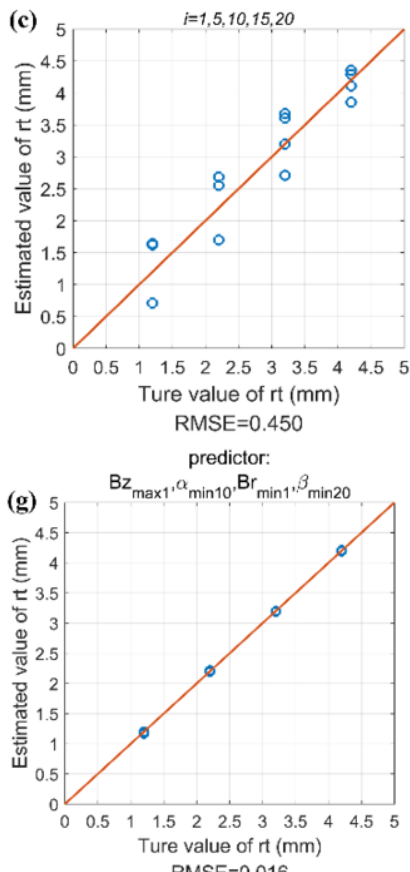

Figure 4. Compare the models with different feature signals as predictors to evaluate rt.

\section{Experimental verification}

\subsection{Experimental setup}

Figure 5 presents the experiment system. The excitation coil was excited by an AC current supplied from the function generator 1 WF1973 (NF Corporation, Yokohama, Japan). A lock-in amplifier 1 LI5640 (NF Corporation, Yokohama, Japan) and a shunt resistor monitor the current and provide a reference frequency to the lock-in amplifier 2 LI5640 (NF Corporation, Yokohama, Japan). Two sensor arraies, each consisting of ten Magneto-Impedance sensors, measure magnetic fields. The signals are measured by the lock-in amplifier 2 and automatically recorded by the PC via the A/D converter. 


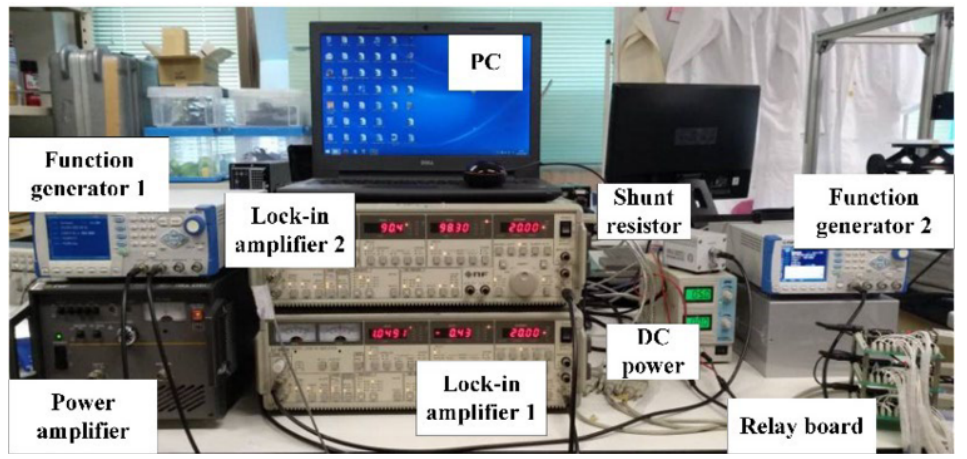

Figure 5. Experiment system.

\subsection{Evaluation of residual wall thickness $r t$}

Figure 6 shows the verification of the evaluation of the prediction model using the experimental signals. As shown in Figure 6(f), the model evaluates best, RMSE $=0.497$. This result is consistent with the experiment, which $\mathrm{Bz}_{\max 1}, \mathrm{Br}_{\min 1}, \alpha_{\min 10}$, and $\beta_{\max 20}$ are the best predictors, the evaluated value of $\mathrm{rt}$ is close to the true value.
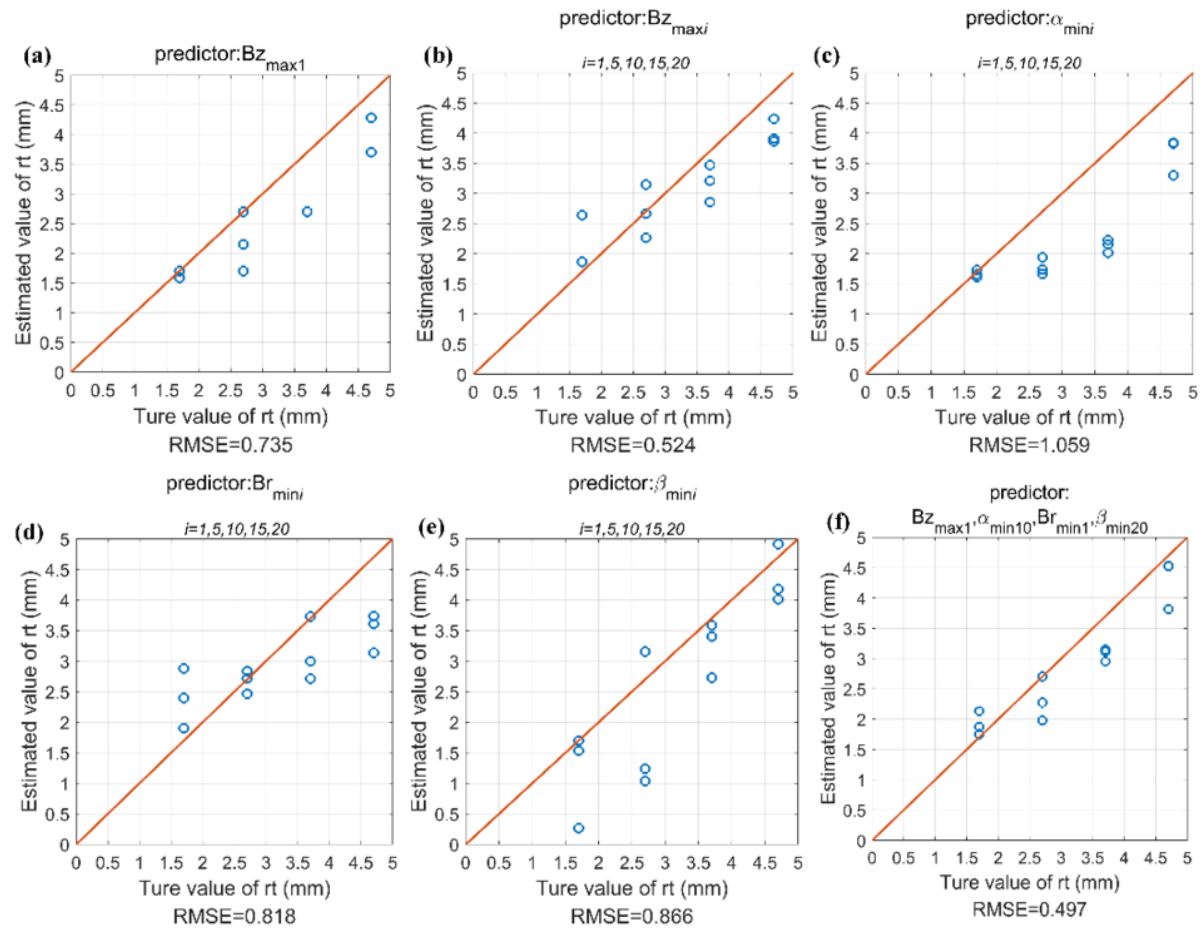

Figure 6. Experimental verification of evaluation of rt. 


\section{Conclusion}

This paper develops the inverse algorithms for evaluating the local wall thinning of carbon steel pipe based on Gaussian process regression. Through comparative analysis, the signal with high correlation coefficient with defect size is used as the predictor, and the evaluation effect of the model is better. The simulation results are basically consistent with the experimental results, indicating the correctness of the simulation, and have a strong guiding role for the actual pipeline inspection.

\section{Acknowledgements}

The work was supported by Key Laboratory of Nondestructive Testing (Nanchang Hangkong University), Ministry of Education under Grant No ZD201629001and Education Department of Zhejiang Province under Grant No Y201534025. The author would like to thank Tohoku University, for sponsoring Ms. Sun's visit to Tohoku University, Japan.

\section{References}

[1] Xie S, Chen Z, Takagi T, Uchimoto T. Quantitative non-destructive evaluation of wall thinning defect in double-layer pipe of nuclear power plants using pulsed ECT method. NDT\&E International. 2015 Oct; 75:87-95.

[2] Hwang KM, Yun H, Seo HK, Lee JY, Kim KW. Development of ToSPACE for pipe wall thinning management in nuclear power plants. 2019;(1):1-5.

[3] Zhdanov AG, Shchukis EG, Lunin VP, Stolyarov AA. Algorithms for pre-processing of eddy-current signals when inspecting heat-exchanger pipes of NPP steam generators. Russian Journal of Nondestructive Testing. 2018 Jun;54(4):283-293.

[4] Evgeny Y, Olga G, Daniil U. Thickness measuring of electroconductive pipe walls using the dualfrequency eddy-current method. 2017 Jan;783(1):012061.

[5] Zhu L. Development and application of eddy current inspection system for heat-exchanging tubes of horizontal steam generator. 2019;40(3):150-154.

[6] Spikic D, Tutic R, Vasic D, Ambrus D, Bilas V. Digital eddy current probe for tube nondestructive testing using binary excitation. IEEE Instrumentation and Measurement Technology Conference. 2019 May;2019: 1091-5281.

[7] Denzel A, Kästner J. Gaussian process regression for geometry optimization. 2018;149(9). 